www.jmscr.igmpublication.org Impact Factor 5.244

Index Copernicus Value: 83.27 ISSN (e)-2347-176x ISSN (p) 2455-0450 crossref DOI: _https://dx.doi.org/10.18535/jmscr/v4i12.11

\title{
Cytopathological Profile of Non Thyroid Neck Swellings - A Tertiary Centre Experience
}

\author{
Authors \\ Anil Kumar Surendran ${ }^{1}$, Arun Punnekkattuchira Rajendraprasad ${ }^{2}$, Meer M Chisthi $^{{ }^{*} *}$ \\ ${ }^{1}$ Additional Professor, Dept of General Surgery, Government Medical College, Trivandrum, Kerala, India \\ ${ }^{2}$ Junior Resident, Dept of General Surgery, Government Medical College, Trivandrum, Kerala, India \\ ${ }^{3}$ Assistant Professor, Dept of General Surgery, Government Medical College, Trivandrum, Kerala, India. \\ Corresponding Author*
}

Dr Meer M Chisthi,

Assistant Professor, Department of General Surgery, Government Medical College,

Trivandrum, Kerala, India 695011

Phone Number 919446560110. Email: meerchisthi@ gmail.com

\begin{abstract}
Background: Neck masses form a relatively common presentation in an Surgical outpatient clinic. In the adult population a neck mass greater than $2 \mathrm{~cm}$ in diameter is reported to have greater than $80 \%$ probability of being malignant. Due to the wide variety of primary sites, good clinical acumen is essential to narrow down the investigations and arrive at a proper diagnosis. The objectives of the study were to find the distribution of benign and malignant lesions among non thyroid neoplastic neck swellings and to describe the demographic profile and other features of these patients.
\end{abstract}

Methods: This was a Descriptive study carried out at the General Surgery department of Government Medical College Thiruvananthapuram. 54 patients with non thyroid neck swellings were evaluated for 1 year and their clinical as well as cytological findings recorded. This data was analysed to find the study objectives.

Results: Among the studied patients, $85.2 \%$ were malignant while 14.8 were benign neoplasms. $78.3 \%$ of malignant neck masses were secondaries and $21.7 \%$ were primary in origin. Pleomorphic adenoma was the commonest benign neoplasm. Histologically $60 \%$ of primary malignant neoplasms turned out to be Hodgkin's lymphoma and $30 \%$ revealed non-Hodgkin lymphoma. Pathology wise, $72.2 \%$ of the cases were squamous cell carcinoma and $13.9 \%$ were adenocarcinoma.

Conclusions: Malignant swellings are the commonest among non thyroid neck masses. Metastatic cervical lymphadenopathy is the predominant pathological type among these swellings. Hence detailed examination and appropriate investigation is essential to localise the primary in time and ensure optimal treatment outcome.

Keywords: Biopsy, Fine Needle Aspiration Cytology, Neck masses, Non thyroid tumors.

\section{Introduction}

A swelling in the neck is very often the first manifestation of an underlying malignancy. Early detection and proper evaluation of swellings that present in this fashion are critical in ensuring adequate outcome. Neck lesions are frequently encountered in clinical practice and found responsible for significant morbidity and mortality across the globe. Common lesions of the neck region include goiters, lymph adenopathy due to 
tuberculosis and other chronic inflammatory lesions, pleomorphic adenoma of salivary glands and cysts. On rare occasions, branchial cysts, thyroglossal duct cysts, carotid glomus tumors, and cysts or tumors of the skin appendages can also be diagnosed. Malignant lesions: primary as well as metastatic, common to the cervical group of lymph nodes, are also frequently noted.

An isolated cervical mass in an adult should be considered malignant until proven otherwise. This statement is derived from 'the Rule of 80s' which states that in adult patients with non thyroidal neck masses, approximately $80 \%$ have neoplasms, $80 \%$ of these are malignant, $80 \%$ among these malignant are metastatic, and $80 \%$ of the primaries are located above the clavicle. This rule is a simplified version of the data collected over 19 years by Gray et al ${ }^{(1)}$. However, this statement comes with a caveat that this rule applies only to masses over $2 \mathrm{~cm}$ in diameter and in patients who are over 35 years of age ${ }^{(2)}$. The rule of $80 \mathrm{~s}$ is a useful rule of thumb especially for physicians not seeing patients with neck masses regularly.

Evaluation of the neck mass itself includes an appropriate assessment of its location, size and consistency. Thorough knowledge of the lymphatic drainage of the salivary glands, thyroid gland, oral cavity and nasopharynx is invaluable for narrowing down the differential diagnoses if there is metastatic nodal disease in specific areas of the neck. The differential diagnosis of a neck mass is usually dependent on its location and patient's age (3). Metastatic squamous cell carcinoma in a cervical lymph node is usually from a primary of the oropharynx, hypopharynx or nasopharynx. Nodal metastasis in the cervical lymph nodes is associated with worse prognosis for most tumour types of the head and neck region (4). Neoplasms of neck region form a big majority of cancers in India, accounting for as high as $23 \%$ of all cancers in males and $6 \%$ in females. The increased prevalence of malignancies in neck region compared to other areas is attributed to the use of tobacco in various forms, poor oral hygiene, and recurrent viral infections ${ }^{(5)}$.
Neck masses are evaluated primarily with imaging and cytology followed by pathological examination. Ultrasound scan forms the initial evaluation modality in most cases. Fine Needle Aspiration Cytology (FNAC) is a useful, minimally traumatic, technique for the initial evaluation of cervical masses but its diagnostic accuracy and value in differentiation of neoplastic (including malignant or benign) and non-neoplastic masses is a highly debated issue. The sensitivity of FNAC in diagnosis of malignant masses is supposed to range from $70 \%$ to $100 \%{ }^{(6)}$. FNAC has a major disadvantage in that it cannot replace histologic examination, as the biological material obtained does not provide precise cellular architectural details. Nevertheless FNAC has the advantage of obtaining samples from multiple lesion sites, thus obviating the need for open biopsy unnecessary in some cases. Ideally, fine-needle aspiration should be performed preoperatively for all cases of doubtful lateral cervical swelling, as the cytological results can direct further investigations.

With this background, this descriptive study was undertaken to find the pattern of non thyroid swellings in a tertiary level institution. The aim of this study was to study the cytological profile of non thyroid neoplastic neck swellings attending the General Surgery department of our institution.

\section{Materials and Methods}

The study setting was the General Surgery Department of our institution, which is the largest tertiary level institution in the public sector and provides health care to large number of patients from the Southern districts of Kerala and Tamilnadu. This study was designed as a Descriptive case study. The study period was for twelve months, from December 2013 to December 2014. The primary objective of the study was to find the distribution of benign and malignant lesions among non thyroid neoplastic neck swellings. Secondary objectives were to find the demographic profile of patients included in the study and to delineate the location of the primary among the metastatic neck masses. 
The study protocol was approved by the scientific committee of the institution followed by Ethics committee. Research subjects included patients with non thyroid neoplastic neck swellings, coming to the General Surgery out Patient department of our institution during the study period who were willing to be included in the study.

Inclusion criteria: Age more than 18 years, swelling size more than $2 \mathrm{~cm}$

Exclusion criteria: Inflammatory swellings, vascular swellings, patients not giving informed consent.

No random sampling technique was used. Every consecutive patient eligible for the study was included. Data collection tool included a structured questionnaire which included the following variables: Patient details: with geographic data, Details of presenting complaints: with duration of swelling, Personal history: smoking, alcohol consumption, other addictions, Family history: malignancy. Clinical examination findings and investigation reports including FNAC and imaging were also recorded.

During FNAC, aspiration was performed by an expert pathologist using a 22 gauge needle and a 10 or 20 milli litre syringe. Two or more specimens were obtained each time. FNAC was performed, and smears were directly prepared for cytology. The aspirated material was spread onto 2 to 4 slides and immediately fixed by immersion in $95 \%$ ethylic alcohol. After fixing with alcohol, smears were stained using Papanicolaou stain. All the patients underwent FNAC along with relevant blood investigations. If history and clinical examination were consistent with lymphoma, excision biopsy of the swelling was done to confirm the diagnosis. Hematoxylin with Eosin staining was used for staining after excision biopsy. Using these data non thyroidal neoplastic neck swellings were categorized based on age, sex, benign or malignant pathology, primary or secondary origin, type of secondary, type of primary etc. Data was recorded into a structured performa and subsequently entered into Microsoft
Excel sheet. All the statistical analyses were performed using Statistical Package for Social Sciences (SPSS Inc., Chicago, Illinois, USA). Data is being presented as mean \pm standard deviation and proportions as appropriate. Descriptive analysis was mostly used for the study. The case with a $p$ value less than 0.05 was considered to be statistically significant as and when relevant.

\section{Results}

During the study period of 1 year, there were 81 patients recruited as study subjects with non thyroid neck swellings fulfilling the inclusion criteria. Out of the 81 cases, 68(83.9\%) were malignant and $13(16.1 \%)$ were benign neoplasms [Table 1]. Among benign neoplasms, pleomorphic adenoma was found to be the commonest pathological type: 5(38.5\%), followed by schwann-oma: $3(23.1 \%)$. In this study it was found that 53(77.9\%) of malignant neck masses were metastatic and only $15(22.1 \%)$ were primary in origin [Table 2]. Primary malignancy was found to occur more in $3^{\text {rd }}$ and $5^{\text {th }}$ decades of life: that is, $30 \%$ each. Histologically, 10 out of the $15(66.7 \%)$ primary malignant neoplasms were Hodgkin's lymphomas, $4(26.7 \%)$ were NonHodgkin lymphoma and $1(6.7 \%)$ was parotid malignancy [Table 3].

In this study, 53 metastatic non thyroid neck masses were found. Out of which $23(43.4 \%$ ) were in the $6^{\text {th }}$ and $21(39.6 \%)$ were in the $7^{\text {th }}$ decade of age. In sex distribution of metastatic neck mass it was found that $64(79 \%)$ were males and $17(21 \%)$ were females. Male: female ratio was found to be 3.76:1. In this study among 53 secondary tumors, primary site was located in 50 cases $(75.5 \%)$, of which 40 cases $(80 \%)$ were of supraclavicular location and 10 cases $(18.9 \%)$ were infraclavicular [Table 4]. Primary site could not be found in 3 cases $(5.7 \%)$. Histological picture of metastatic non-thyroid neck masses showed that 39 cases (73.6\%) were squamous cell carcinoma, $8(15.1 \%)$ adenocarcinoma and 6(11.3\%) of undifferentiated histology [Table 5]. 
Table 1. Distribution of benign and malignant swellings

\section{Number of patients}

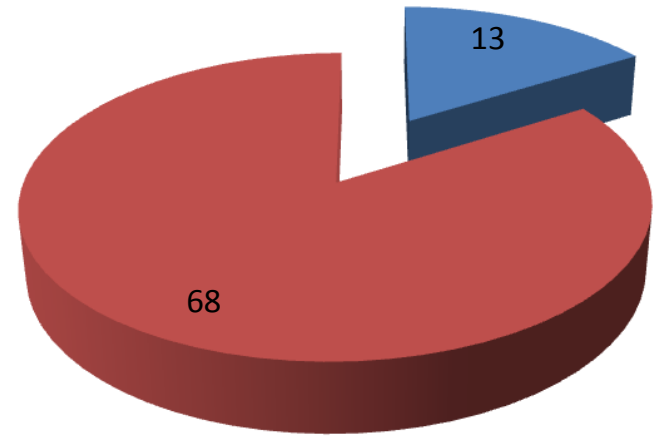

- Benign

Malignant

Table 2. Distribution of primary and secondary among malignancies

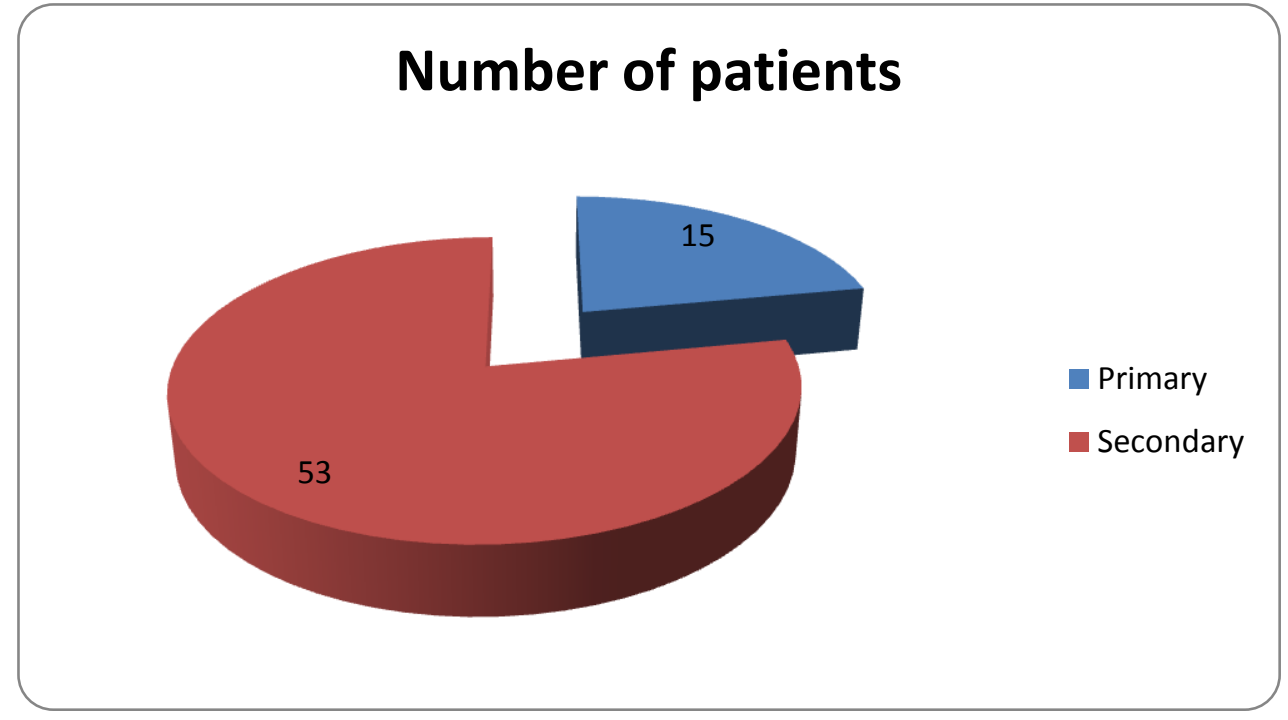

Table 3. Distribution of primary malignancy

\section{Number of patients}

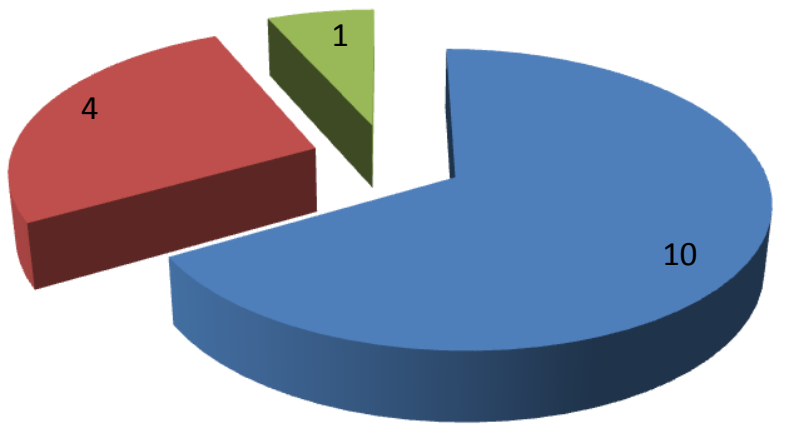

- Hodgkin's lymphoma

- Non Hodgkin's lymphoma

Parotid 
Table 4. Location of primary in metastatic swellings

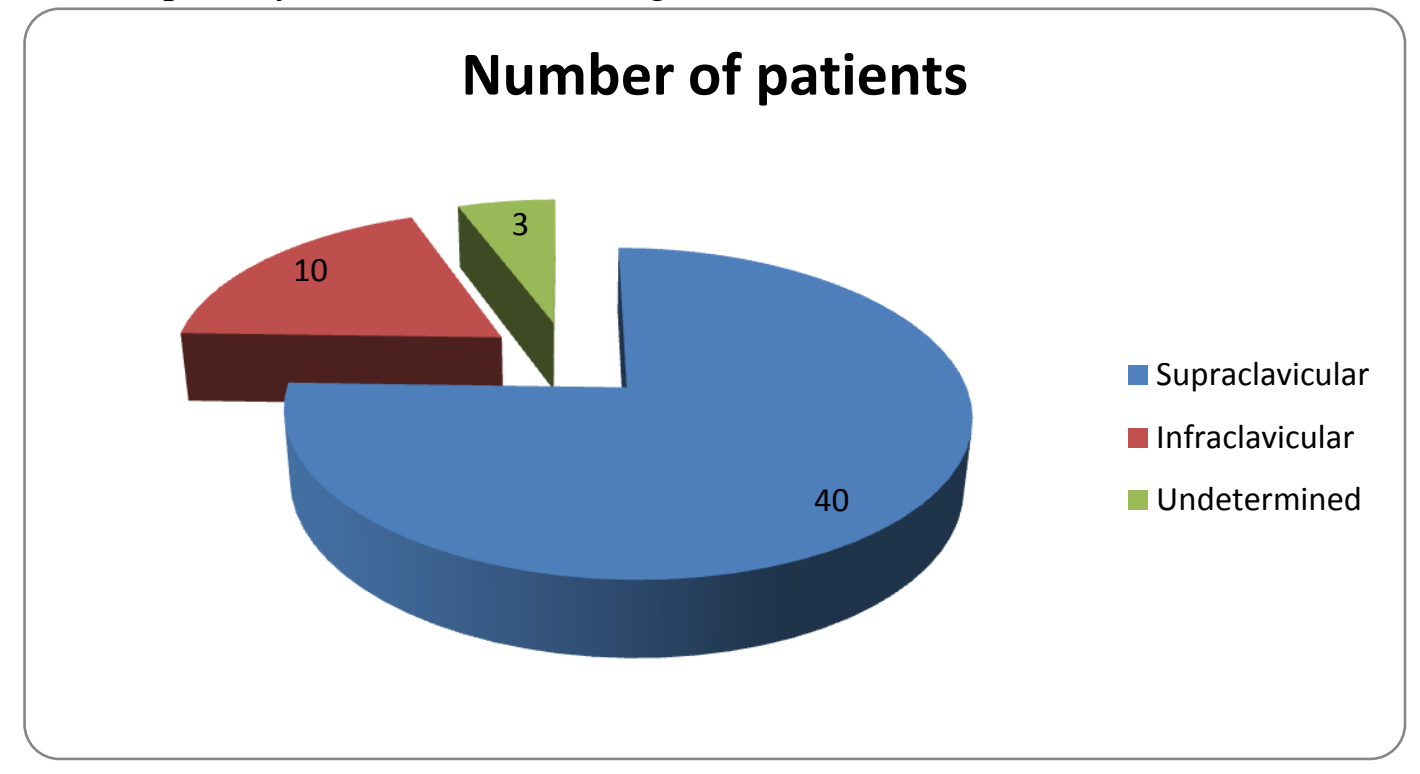

Table 5. Histological types of metastatic swellings

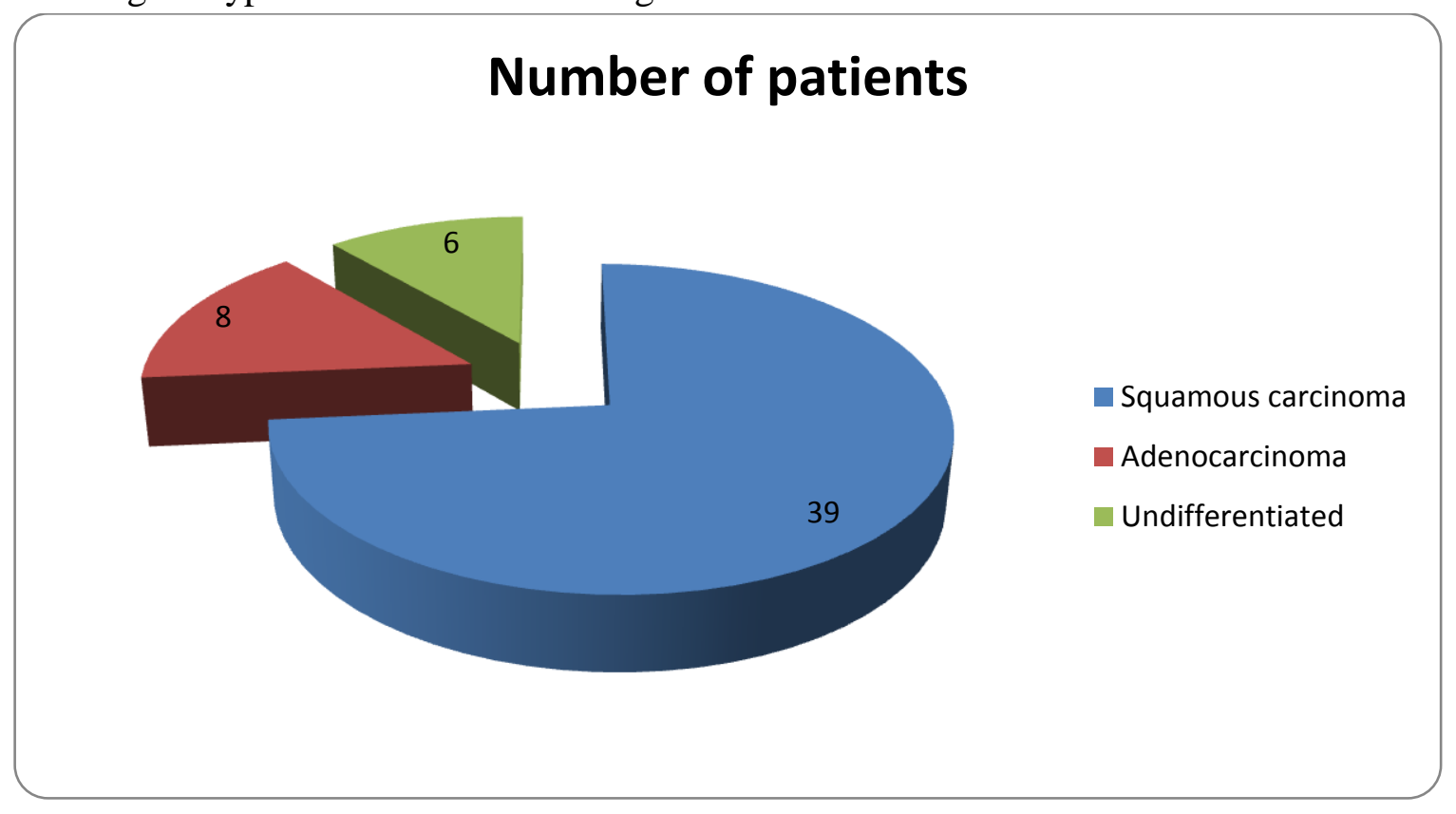

\section{Discussion}

Non thyroid neck mass are a common problem in everyday surgical practice. In this study we considered 81 cases of which $16.1 \%$ of the cases were benign and $83.9 \%$ of the cases were malignant neoplasms. Among the malignant neoplasms, $77.9 \%$ were found to be metastatic and the rest primary lesion. According to a study by Way et al ${ }^{(7)} 15 \%$ of neoplastic non thyroid neck mass were benign and $85 \%$ were malignant, and among malignant non-thyroid neck swelling $85 \%$ were metastatic and $15 \%$ primary lesion.
Only 13 non thyroid benign neck masses were encountered in this study. Of these 13 patients, 5 cases $(38.5 \%)$ were pleomorphic adenomas, 3 (23.1\%) schwannomas and 2(13.3\%) were lipoma, and $1(6.7 \%)$ each was fibroma and angiofibroma. Solom and his associates ${ }^{(8)}$ in their study of 288 cases reported that $18.8 \%$ of benign swellings were pleomorphic adenomas and only $1.04 \%$ were lipomas. The variation may be due to the small number in this series.

In analyzing the age incidence it was found that primary malignant swellings were more common 
in younger age group, that is, the $3^{\text {rd }}$ and $5^{\text {th }}$ decade while the metastatic swellings were common in the $6^{\text {th }}$ and $7^{\text {th }}$ decades. Secondary tumors were more common in males, male female ratio being 3.76:1. This ratio is similar to other studies: 4:1 in a study by Maran ${ }^{(9)}$ and 4.73:1 in a study by Wizenbergh et al ${ }^{(10)}$. In case of primary malignant neoplasm the ratio was 1.5:1. Way et al (7) described that tobacco and alcohol are the most important etiological factors in head neck cancer. Incidence of tobacco consumption is definitely more in Indian population, especially among males.

In this study $66.7 \%$ of primary neoplasm in the neck were found to be Hodgkin's lymphoma, 26.7\% were Non Hodgkin lymphoma and 6.7\% were parotid malignancies. Russell et al ${ }^{(11)}$ also described Hodgkin's lymphoma as the commonest type of primary in the neck. It was found that $75.5 \%$ of the secondary tumor in the neck arose from supraclavicular primary sites, $18.9 \%$ from infraclavicular site and $5.7 \%$ from undetermined origin. Way et al ${ }^{(7)}$ described that $85 \%$ of secondary tumor originated from primary above clavicle and $15 \%$ below the clavicle. These findings are similar to what we found out. Due to limitations in investigations, we could not find out the primary sites of $5.7 \%$ of cases. Histologically it was found that $75 \%$ cases were squamous cell carcinoma, $14.7 \%$ were adenocarcinoma and $10.3 \%$ undifferentiated. Rahman and his study group ${ }^{(12)}$ found that $70.6 \%$ were squamous cell carcinoma, $23.5 \%$ undifferentiated carcinoma and $5.9 \%$ were adenocarcinoma. In our study incidence of undifferentiated carcinoma was lower and that of adenocarcinoma slightly higher than the study of Rahman ${ }^{(12)}$.

\section{Conclusions}

To summarize, malignant swelling is the commonest non thyroid neoplastic neck mass among Indian patients. Also, metastatic cervical lymphadenopathy is the most frequent in the study context. Pleomorphic adenoma is the commonest benign neoplasm while Hodgkin's lymphoma is the commonest malignant primary in the neck. Pathologically, majority of the secondaries are from squamous cell carcinoma.

The limitations of the study include the fact that the research being tertiary care centre based, may not be representative of the actual situation in the community. Also, there is an apparent difficulty in quantifying the burden of the disease because neck swelling also go to other departments in the institution itself including ENT, OMFS and Medical Oncology as well as to Regional Cancer Centre which is a specialized cancer research center within the vicinity of our institution.

In conclusion, metastatic lymphadenopathy is the commonest neoplastic swelling in the neck apart from thyroid gland swellings. For the proper management of neck masses, early diagnosis and timely intervention is essential. Hence it is essential that diagnostic facilities be adequately modernized and susceptible population be investigated promptly.

\section{Acknowledgements}

The authors are thankful to Dr. Sreekumar A, formerly Professor and Head, Department of General Surgery, Government Medical College, Trivandrum, for his guidance during the course of the study.

\section{References}

1. Gray SW, Skandalakis JE, Andrulakis JA, Non thyroid tumour of the neck. Contemp. Surg. 1985, 26: 13-24.

2. Scott -Brown's Otorhinolaryngalogy, Head and Neck Surgery. $7^{\text {th }}$ Edition, $3^{\text {rd }}$ Volume, Chapter 140,Page 1777

3. Davenport M. Lumps and swellings of the head and neck.BMJ 1996;312:368-371.

4. Townsend J, Courtney M. Editor in chief. Sabiston Textbook of Surgery: The Biological Basis of Modern Surgical Practise. $16^{\text {th }}$ Ed. Harcourt Asia Ptc Ltd, W B Saunders Company. 2001; 546

5. Mehrotra R, Singh M, Kumar D, Pandey AN, Gupta RK, Sinha US. Age specific 
incidence rate and pathological spectrum of oral cancer in Allahabad. Indian J Med Sci. 2003;57:400-4.

6. Saatian M, Badie BM, Shahriari S, Fattahi F, Rasoolinejad M. FNA diagnostic value in patients with neck masses in two teaching hospitals in Iran. Acta Med Iran. 2011;49(2):85-8.

7. Way LW, Doherty GM. Editor. Current Surgical Diagnosis and Treatment, 11 ed. USA Lange Medical Books/ Mc Graw Hill.2003 282-283.

8. Solom BS, Schnder EE. Differential Diagnosis of a mass in upper lateral neck. J Laryngol Otol. 1981;95:104

9. Maran ADG. Editor. Login Turners Disease of the Nose Throat and Ear, $10^{\text {th }}$ ed. Wright. $1980 ; 202$.

10. Wizenberg MJ, Bloedorn FG, Winster S, Gracia J. Treatment of Lymphnode Metastasis in Head and Neck cancer. Cancer, 1972;29:1456

11. Russel RCG, Williams NS, Bulstrnde C J $\mathrm{K}$, Editors. Bailey and Love's Short Practice of Surgery, 24th ed London, Arnold.2004;756

12. Rahman SM. Non - thyroid Neck Swelling in adult; A clinicopathological study of 50 cases (Dissertion).Dhaka; Bangladesh College of Physicians and Surgeons.1987;70-4 